\title{
When do armed revolts succeed: lessons from Lanchester theory
}

\author{
MP Atkinson ${ }^{1}$, A Gutfraind ${ }^{2 *}$ and M Kress ${ }^{1}$ \\ ${ }^{1}$ Naval Postgraduate School, Monterey, CA, USA; and ${ }^{2}$ The University of Texas at Austin, Austin, TX, \\ USA
}

Major revolts have recently erupted in parts of the Middle East with substantial international repercussions. Predicting, coping with and winning those revolts have become a grave problem for many regimes and for world powers. We propose a new model of such revolts that describes their evolution by building on the classic Lanchester theory of combat. The model accounts for the split in the population between those loyal to the regime and those favouring the rebels. We show that, contrary to classical Lanchesterian insights regarding traditional force-on-force engagements, the outcome of a revolt is independent of the initial force sizes; it only depends on the fraction of the population supporting each side and their combat effectiveness. The model's predictions are consistent with the situations currently observed in Afghanistan, Libya and Syria (September 2011), and it points to how those situations might evolve.

Journal of the Operational Research Society (2012) 63, 1363-1373. doi:10.1057/jors.2011.146

Published online 21 December 2011

Keywords: conflict analysis; defence studies; system dynamics; population; behaviour

\section{Introduction}

Recent (2011) events in Libya underscore the significant impact of armed revolts on regional and global interests. Armed revolts typically start with demonstrations and civic unrest that quickly turn into local violence and then fullscale combat. (The terms revolt, rebellion, and insurgency are interchangeable in most senses and we use the term revolt throughout for consistency.) As demonstrated in Libya, the evolution of the armed revolt has a strong spatial component; individuals in some regions (eg, parts of Tripoli) may be loyal to the regime because of ideology or economic and social incentives or fear, whereas other regions (eg, Benghazi) become bastions of the rebels powered by strong local popular support. Thus, armed revolts, very much like conventional war, are about gaining and controlling populated territory. However, unlike conventional force-on-force engagements, where the civilian population plays a background role, armed revolts are characterized by the active role of the people, who become a major factor in determining the outcome of the conflict: both the rebels and the regime need the support of the population to carry out their campaigns (Lynn, 2005; Hammes, 2006).

\footnotetext{
${ }^{*}$ Correspondence: A Gutfraind, Center for Computational Biology and Bioinformatics, The University of Texas at Austin, 1 University Station C0930, Austin, TX 78712, USA.

E-mail: agutfraind.research@gmail.com
}

Armed revolts differ from civil uprisings, such as those that occurred recently (2011) in Tunisia and Egypt, because uprisings are manifested in demonstrations, which may involve some minor local violence, rather than protracted armed engagements. Armed revolts often originate from civil uprisings and thus our model may provide insight into the situation if the uprising is on the verge of evolving into the more violent revolt. An example of this possible progression can be found in Syria (September 2011) where an armed revolt seems to be emerging from a popular uprising mostly owing to defections from the government forces and some foreign supply of arms to local organizations.

Our approach to modelling armed revolts is on the basis of Lanchester theory (Lanchester, 1916) that describes the strength of two opposing military forces by two ordinary differential equations (ODEs). The forces cause mutual attrition that depletes their strengths until one of the forces is defeated. Although Lanchester models are stylized and highly abstract, they have been extensively used for analysis for almost a century because they provide profound insights regarding conditions that affect the outcomes of military conflicts. Examples of such analysis using Lanchester models include studies of the Battle of Britain (Johnson and MacKay, 2011), the Battle of Kursk (Lucas and Turkes, 2004), the Ardennes Campaign (Hung et al, 2005), the Battle of Iwo Jima (Engel, 1954), and the Battle of Inchon (Hartley and Helmbold, 1995). For further information on the analysis and applications of Lanchester models please refer to Washburn and Kress (2009). 
In the 1960s, Deitchman (1962) and Schaffer (1968) proposed variants of the classic Lanchester equations that more accurately model guerrilla and insurgent warfare. The key observation is that attrition of the guerrillas depends not just on the government forces, but also on the size of the guerrilla force. The smaller the guerrilla army, the more difficult it is for government forces to target them. Later works examined more directly the role of the population in insurgency conflicts. Intriligator and Brito (1988) explicitly modelled the level of popular support for the insurgents with an additional state variable. McCormick and Giordano (2002) considered only two state variables but introduced terms that allow the government and insurgents to increase in strength on the basis of popular support. Kress and Szechtman (2009) analysed the impact of the population by focusing on how collateral damage and intelligence alter the dynamics. Blank et al (2008) used Lanchester techniques to study Operation Iraqi Freedom.

Here we also examine a conflict between two forces where the population plays an important role. However, unlike the traditional Lanchester models used by the previously cited works, our focus is not on attrition but on the control of friendly and hostile territories. Using our model, we derive the end-state of the revolt, identify stalemate situations and study the effects of foreign intervention and of inconstant support by the population. We show that contrary to classical Lanchesterian insights regarding traditional force-on-force engagements, the outcome of a revolt is independent of the initial force sizes. We also derive conditions for successful foreign interventions.

The main contribution of our model is methodologicalextending the classical Lanchester theory to armed revolts where the population is a key player. Although we dare to make some predictions (about Syria), this is not the main objective of our work. Similarly to classical Lanchester models, our model is not predictive; it is descriptive and, at best, diagnostic. Its purpose is to gain insights about causeand-effect relations. The main reason for this restrictive objective is the lack of relevant and reliable data. Having said that, using the little data available we note that the results of the model are consistent with the situations currently (September 2011) observed in Afghanistan, Libya and Syria. We also evaluate policy options facing the international community.

\section{Setting and assumptions}

Consider an armed revolt involving two forces, termed Red and Blue, that rely on the population for manpower, intelligence, and most other resources. In most situations one of these forces will be in the position of power (eg, the government forces) and may hold an advantage. We assume a polarized situation where there are no neutrals in the population that is divided into supporters of
Blue, called henceforth supporters, and supporters of Red, called henceforth contrarians. We initially assume that the support strongly depends on factors such as tribal affiliation, social class, and ideology and therefore remains unchanged during the armed revolt. However, later on we relax this assumption and allow for changes in popular behaviour, reflecting pragmatic and opportunistic responses of the population to changes in the force balance.

We assume that the country is divided between Red and Blue and therefore a populated region lost by one force is gained by the other force. Independent or neutral regions are not considered because individuals in these areas will often become entangled in the conflict, even if they initially do not want to, and will eventually support one of the sides. We also assume that the population in each region is homogeneous - either supporters or contrarians. This assumption is reasonable if the resolution of the regions is high enough, and is valid in particular in tribal societies where members of a clan closely follow their leader.

A force that fights over a region might be either supported or opposed by the local population, situations that we call liberation or subjugation, respectively. A liberating force fights more effectively than a subjugating force because of population support, Ceteris paribus. Moreover, the forces in control of hostile regions are busy policing the population and therefore adopt a defensive posture. Thus, only the forces operating in friendly regions proactively attempt to capture additional territories.

\section{Model}

Let $S$ and $C(S+C=1)$ denote the fraction of the total population who are supporters of Blue and supporters of Red ('contrarians'), respectively. Let $B$ and $R(B+R=1)$ denote the fraction of the population controlled by Blue and Red, respectively. We use the notation $X Y$ for the fraction of population $X$ that is controlled by force $Y$, where $X=S, C$ and $Y=B, R$. Hence, $S B+S R=S$ and $C B+C R=C$. The offensive strengths of the Blue and Red combatants are proportional to $S B$ and $C R$, respectively. When Blue subjugates a $C R$ region it becomes part of $C B$ and when Blue liberates an $S R$ area it becomes part of $S B$. Similar actions are possible by Red, giving a total of four kinds of combat engagements, as shown in Figure 1. We implicitly assume that the country can be divided into areas or regions of sufficiently small size so that each one can be viewed as a homogeneous group of people belonging to one of the four types.

Because Red and Blue operate in populated areas, the outcome of an engagement depends both on the strength of the attacking force but also on the signature (ie, visibility) of the defending force; smaller attack force (fewer shooters) or smaller signature (fewer targets) result in a smaller gain/ loss rate. Namely, at each interaction, the gain rate of the 


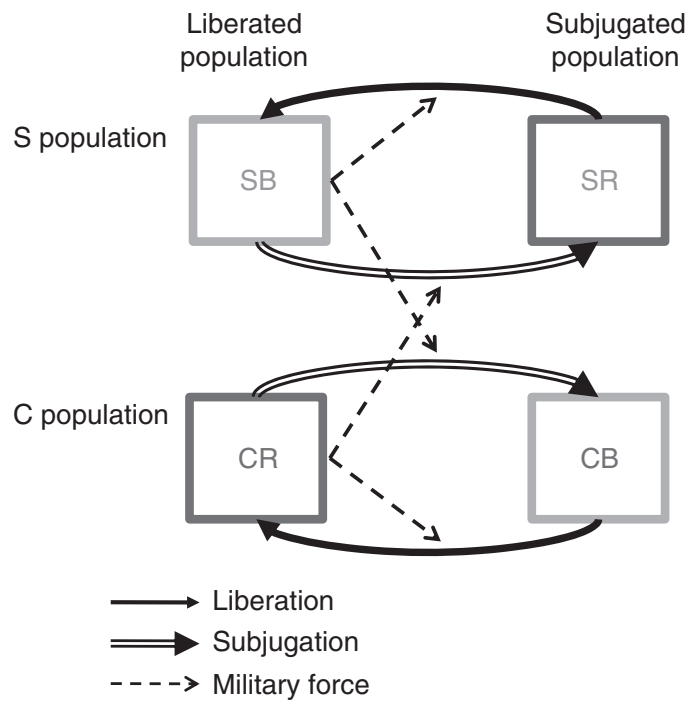

Figure 1 Schematic dynamics of the model. The four variables in the model appear as boxes, where each box represents a possible combination of population behaviour and controlling force. Solid lines indicate change in control of population although dashed lines indicate the force causing it. Observe that the population does not change allegiances even under occupation.

attacker is given by a scaling constant, called henceforth attrition rate, multiplied by the product of the attacking and defending force sizes. This relationship implies that even a large attacker would struggle to find and suppress a small defender (or insurgent) diffused in the population. The resulting model is an adaptation of the Lanchester Linear Law (see eg, Washburn and Kress, 2009, p 83) and Deitchman's guerrilla warfare model (Deitchman, 1962).

The attrition rate constants depend on the tactics, technology, and equipment of the parties. Thus if one of the sides represents the government, which will have an initial advantage in many of these categories, these attrition rates will capture this advantage. The attrition rates, however, also depend upon the behaviour of the population. Thus, let $f_{S}$ and $f_{C}$ denote the rates of liberation of friendly regions by Blue and Red forces, respectively. Similarly, let $h_{C}$ and $h_{S}$ denote the rates of subjugation of hostile regions by Blue and Red, respectively. The resulting dynamics is given in Equation (1):

$$
\begin{aligned}
& S B^{\prime}=+f_{S} S B \cdot S R-h_{S} C R \cdot S B \\
& S R^{\prime}=-f_{S} S B \cdot S R+h_{S} C R \cdot S B \\
& C R^{\prime}=+f_{C} C R \cdot C B-h_{C} S B \cdot C R \\
& C B^{\prime}=-f_{C} C R \cdot C B+h_{C} S B \cdot C R
\end{aligned}
$$

As it is easier to fight in friendly territory, we make the following dominance assumption:

$$
f_{S}>h_{C} \text { and } f_{C}>h_{S}
$$

\section{End-state of the revolt}

From solving Equation (1) we obtain that the conflict can result in one of three outcomes, corresponding to the stable equilibrium points of the equations:

1. Blue victory: $S B+C B=1$,

2. Red victory: $C R+S R=1$,

3. Stalemate: Both sides control a fraction of the total population.

It can be shown that the evolution of the conflict does not involve cycles where populated regions change sides endlessly; rather, the conflict dissipates and reaches a stable state. Proofs of this and all other results are given in the Appendix at the end of this paper.

The stable outcomes are not dependent on all four attrition rates but rather on two ratios: $r_{S}=f_{S} / h_{S}$ and $r_{C}=f_{C} / h_{C}$. We call these the 'liberation-subjugation effectiveness ratio' (LSER) of supporters and contrarians, respectively. These ratios account for differences in tactics, technology, and information between Blue and Red, and also reflect the ability and commitment of the local population to support its preferred force. The outcomes are

$$
\begin{array}{ll}
\text { Blue wins if and only if } & r_{C}<\frac{S}{1-S} \\
\text { Red wins if and only if } & r_{S}<\frac{1-S}{S}
\end{array}
$$

Otherwise a stalemate occurs.

These results ${ }^{1}$ are summarized in Figure 2(a). It follows from Equations 3 to 4 that the fate of the armed revolt is completely determined by the LSERs and the population split between supporters $S$ and contrarians $C=1-S$; it does not depend on the initial sizes of the Blue and Red forces. Moreover, the minimum popular support needed to guarantee Blue's win only depends on the LSER in the contrarians' territory. Specifically, Blue wins if and only if $r_{C}(1-S)<S$, that is, if the fraction of its supporters is larger than the fraction of contrarians times the LSER in contrarians' territory. An equivalent statement applies for Red victory that happens if and only if $r_{S}(1-C)<C$. The operational implication of these two conditions is that strengthening one's advantage in friendly territories (eg, Blue increasing $r_{S}$ ) may be sufficient to avoid defeat but not to secure a win; if one is not effectively fighting in hostile territory (eg, Blue cannot sufficiently decrease $r_{C}$ )

\footnotetext{
${ }^{1}$ Technically, we assume that at the start of the dynamics both forces have some presence in a friendly territory, ie $S B_{0}>0$ and $C R_{0}>0$. Otherwise, one of the forces is never challenged and wins trivially. Also, the model has a fourth equilibrium that corresponds to the case where the territory is divided between Blue and Red who control only hostile territory $(S R+C B=1)$. Obviously, such a situation is very unlikely and indeed this equilibrium is unstable, as shown in the Appendix.
} 

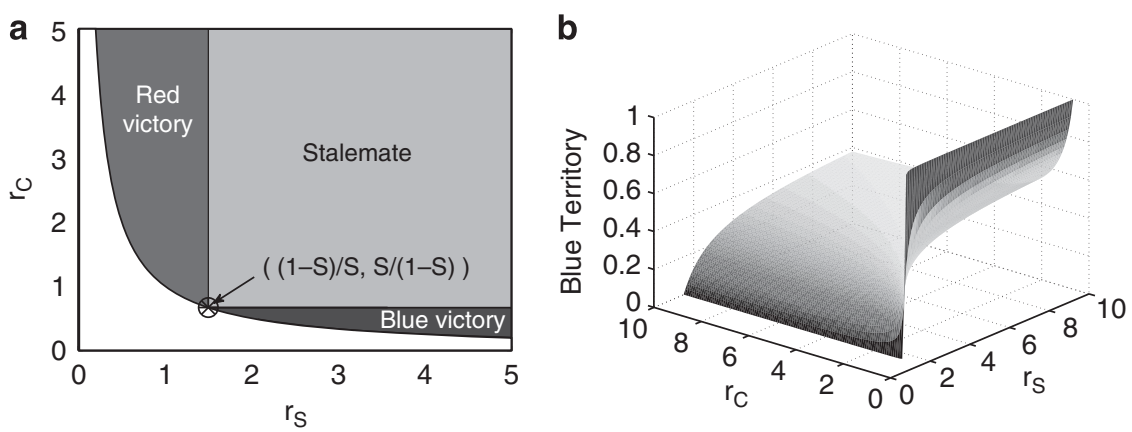

Figure 2 Outcomes of the conflict when $S=40 \%$ as a function of $r_{S}$ and $r_{C}$. (a) The possible outcomes are: stalemate, Red victory and Blue victory. The white area is excluded by the dominance assumption (In Equation 2). Outright victory is possible only when one party has a low LSER. Increasing $r_{S}$ and $r_{C}$ makes both parties much more entrenched in their areas, leading to a stalemate regardless of the value of $S$. (b) The amount of territory controlled by Blue. Observe that a very sharp change in the outcome is predicted as $r_{C}$ approaches $2 / 3$, from a balanced stalemate to a Blue victory.

then one cannot win; the best it can hope for is a stalemate. At the stalemate equilibrium, denoted $X Y_{b}$

$$
\begin{array}{ll}
C B_{b}=\frac{S\left(1+r_{S}\right)-1}{r_{S} r_{C}-1}, & S R_{b}=\frac{r_{C}-S\left(1+r_{C}\right)}{r_{S} r_{C}-1}, \\
S B_{b}=r_{C} C B_{b}, & C R_{b}=r_{S} S R_{b} .
\end{array}
$$

Notice that the denominators are always positive because of the dominance assumption (see Equation 2). Equation (5) indicates that as $S$ increases an increasing part of the population is controlled by Blue. When $r_{C}$ increases, a larger fraction of the contrarians is able to remain free (ie, ruled by Red).

We plot the fraction of the population controlled by Blue during a stalemate (ie, $C B+S B$ ) in Figure 2(b). (We only present the plot for $S=0.4$, but other values of $S$ are qualitatively similar.) Near the Blue victory condition defined by Equation 3 the fraction of the population controlled by Blue is near one, but quickly decreases as $r_{C}$ increases. Similarly, the fraction of population controlled by Blue rapidly increases as $r_{S}$ moves away from the Red victory condition. However, after the significant initial change in the fraction of Blue's regions as $r_{S}$ or $r_{C}$ increases, the surface flattens out. As both $r_{S}$ and $r_{C}$ continue to increase, the fraction of Blue's regions approaches $S$. Therefore, when $r_{S}$ and $r_{C}$ are reasonably bounded away from their thresholds, an entrenched stalemate occurs where Red and Blue control primarily their friendly territories.

\section{Extensions of the basic model}

We consider now two extensions of the basic model: the case of foreign intervention and the case of shifting popular support.

\section{Foreign military intervention}

Most large revolts in modern times involved foreign military interventions by regional or global powers (Small and Singer, 1982; Sarkees and Wayman, 2010). Such interventions can be manifested in two ways: direct and indirect. Direct intervention (eg, air-strike support to ground units, such as the intervention of NATO forces in Libya in 2011) allows the supported side to exercise more firepower against its opponent. Indirect intervention provides the supported side with force multipliers such as intelligence, training, logistical support and advanced weapons, but no additional firepower per se. In both cases we assume that just one side, say Blue, receives the foreign support. We leave for future studies to consider the case of foreign support to both sides.

\section{Direct intervention}

For simplicity, suppose that the foreign constituent is tactically superior and it experiences negligible attrition (eg, air support for Blue that is subject to ineffective air defense of Red). Therefore, the effectiveness of the foreign constituent remains fixed throughout the armed revolt. However, similarly to the direct engagements discussed above, its ability to target Red diminishes as the size of Red's forces decreases. In that case, Red targets are harder to find and engage. Let $\lambda_{S}, \lambda_{C}>0$ denote the combat power of the foreign constituent when operating in supporters' $(S)$ regions and contrarians' $(C)$ regions, respectively. The separation into two combat power parameters allow for the possibility that the foreign constituent only contributes to certain kinds of operations (eg, only to liberating supporters), and/or is affected by the behaviour of the population, just like Blue. In this case, Equation (1) becomes

$$
\begin{aligned}
& S B^{\prime}=+f_{S} S B \cdot S R-h_{S} C R \cdot S B+\lambda_{S} S R \\
& S R^{\prime}=-f_{S} S B \cdot S R+h_{S} C R \cdot S B-\lambda_{S} S R \\
& C R^{\prime}=+f_{C} C R \cdot C B-h_{C} S B \cdot C R-\lambda_{C} C R \\
& C B^{\prime}=-f_{C} C R \cdot C B+h_{C} S B \cdot C R+\lambda_{C} C R
\end{aligned}
$$

As the effectiveness of the foreign constituent remains unaffected, it is clear that Red cannot win. The only two 
outcomes are Blue's victory and a stalemate. Blue wins if and only if $\lambda_{C}>f_{C}(1-S)-h_{C} S$. Otherwise, the armed revolt ends in a stalemate. Like in the basic model, the conflict dissipates and reaches a stable state, and no cycles are possible.

An interesting observation is that the value of $\lambda_{S}$ the combat power of the foreign constituent in friendly regions - plays no role in helping Blue achieve victory; it only ensures that Blue will not lose as long as $\lambda_{S}>0$. The threshold of $\lambda_{C}$ that determines Blue's victory is the difference between two terms, each a combination of combat effectiveness and popular support: $f_{C}(1-S)$ is Red's effectiveness fighting on friendly territory times its popular support, and $h_{C} S$ is Blue's effectiveness fighting on hostile territory times its popular support. Clearly, this threshold decreases as the support to Blue increases. In particular, a sufficient condition for Blue victory is $\lambda_{C}>f_{C}$ that only depends upon the fighting effectiveness of Red. Consequently, even if Blue has limited tactical capabilities or a small amount of popular support, it can still prevail with enough assistance from a foreign constituent.

We do not consider the situation where the foreign constituent experiences attrition. The standard example would be foreign ground troops participating directly in combat, such as the US involvement in Vietnam. Analysing this scenario would involve defining an additional state variable for the strength of the intervening force and modelling its dynamics (eg, attrition and reinforcements). Such an expanded model is beyond the scope of this paper.

\section{Indirect intervention}

Indirect intervention (force multiplier) increases the ability of Blue to defend its territory and to attack Red forces. Specifically, the liberation rate $f_{S}$ and the subjugation rate $h_{C}$ are multiplied by factors $\mu_{S}, \mu_{C}>1$, respectively, where the structure of Equation (1) remains unchanged. The LSER values $r_{S}$ and $r_{C}$ change to $\mu_{S} r_{S}$ and $r_{C} / \mu_{C}$, respectively. Using the conditions in Equation 4 we obtain that for Blue to avoid defeat it is sufficient that the intervention be such that

$$
\mu_{S} \geqslant \frac{1-S}{r_{S} S} .
$$

We see that if the Blue forces have low LSER, ie, cannot hold their own territory, they may be defeated despite assistance from their foreign backers. In order to secure a win, it follows from Equation 3 that the support for Blue must be such that

$$
\mu_{C}>\frac{r_{C}(1-S)}{S}
$$

Because $r_{S} r_{C}>1$, the threshold of $\mu_{C}$ is always larger than the threshold of $\mu_{S}$ it is more costly to secure a victory than to avoid a loss. Obviously, the indirect intervention is needed to secure a victory only if $S$ is small enough, specifically, if $S<\left(r_{C}\right) /\left(1+r_{C}\right)$. Note that 'small enough' may actually be quite large when Red is very effective on its own turf compared with Blue ( $r_{C}$ is large).

\section{Opportunistic population}

Although in some conflicts the behaviour of the people is highly polarized and unchanging, in others the population might be quite opportunistic and favour the side that appears more likely to win. It follows that the fraction of the supporters, and hence contrarians, changes according to the state of the conflict. We capture this situation by treating the fraction of supporters $S$ as a dynamic variable, and adding to Equation (1) an equation for $S^{\prime}$. The value of $S^{\prime}$ increases with the fraction of population Blue controls $(S B+C B)$ and decreases with the fraction controlled by Red $(C R+S R)$. Because $C=1-S, S R=S-S B$, and $C B=1-S-C R$, we obtain from Equation (1) the three equations:

$$
\begin{aligned}
S B^{\prime}= & +f_{S} S B(S-S B)-h_{S} C R \cdot S B \\
C R^{\prime}= & +f_{C} C R(1-S-C R)-h_{C} S B \cdot C R \\
S^{\prime}= & +\alpha(S B+1-S-C R)(1-S) \\
& -\alpha(C R+S-S B) S,
\end{aligned}
$$

where $\alpha$ is a parameter that determines the rate at which individuals switch allegiances, which is assumed to be the same for both the supporters and contrarians. With opportunistic population there are only two potential outcomes:

1. Blue victory where the entire population supports Blue, who controls all regions $(S B=1)$, and

2. Red victory where the entire population supports Red, who controls all regions $(C R=1)$.

These two equilibria are stable for all parameter values. There are also two stalemate equilibria: a balanced stalemate where $S B, C R>0$, and a disarmed stalemate where $S B=C R=0$. Neither of the two stalemate equilibria are stable. The disarmed stalemate is neither realistic nor relevant. The balanced stalemate, given below, is more interesting because it lies on a boundary that separates the basins of attraction for the two victory situations:

$$
\begin{aligned}
S B^{*} & =\frac{r_{C}}{2+r_{S}+r_{C}}, \\
C R^{*} & =\frac{r_{S}}{2+r_{S}+r_{C}}, \\
S^{*} & =\frac{1+r_{C}}{2+r_{S}+r_{C}} .
\end{aligned}
$$


Thus, the stalemate equilibrium gives a rough metric for the potential outcome of the conflict. For example, the closer the stalemate equilibrium is to the Blue victory point, the more likely Red will win the conflict. This occurs because most of the three-dimensional $\left(r_{S}, r_{C}, S\right)$ parameter space lies in the basin of attraction corresponding to Red victory.

\section{Comments on recent revolts}

In this section we discuss several nascent and ongoing (in 2011) revolts in light of the $S B C R$ model above and its extensions. We have also attempted to systematically validate this model empirically, but while data on armed conflicts exist (eg, UCDP/PRIO Armed Conflict Dataset (Themnér and Wallensteen, 2011) and Correlates of War Data (Sarkees and Wayman, 2010)), none of the data sets include the information necessary for us to make quantitative estimates of the parameters, particularly the parameters $r_{S}$, and $r_{C}$. Thus, although we could not compute precise estimates of the model parameters, we show that many of the ongoing conflicts are at a state consistent with our model. The model suggests how the outcomes might be effected by decisions including those currently on the policy table.

\section{Libya}

The available information regarding the revolt in Libya is based mainly on fragmented, and largely anectodal, news reports. It suggests that the conflict has progressed through three stages: During the first stage, mid-February to mid-March 2011, the rebels established local governments but then experienced setbacks and were collapsing in the face of a regime offensive towards their base in Benghazi (MacAskill et al, 2011). In the second stage, midMarch to late April, NATO established a no-fly zone and blocked further incursions by Qaddafi's forces. A stalemate developed with the rebels repelling Qaddafi's forces and vice versa. Finally, in the third stage, from early May to September, NATO gradually intensified its air strikes and aid to the rebels leading to rapid rebel progress, and eventually to the fall of Tripoli.

This progression is consistent with our model, as follows. In the first stage, Qaddafi's forces (labelled Red) were much better trained, equipped and organized than the rebels (labelled Blue). The regime's forces could successfully beat the rebels even in rebel-supporting regions, suggesting that $f_{S}<h_{S}$ and $f_{C}>h_{C}$, which implies that $r_{S}<1$ and $r_{C}>1$. From Equation 4 and that $S \approx 40 \%$ we learn that Qaddafi should have achieved a clear victory, crushing the revolt. (Here we estimated $S$ from the fact that three of the seven largest districts in Libya-Benghazi, Misrata, and Az-Zawiya - established rebel governance, and these amount to approximately $40 \%$ of Libya's population.)
In the second stage, the foreign intervention was defensive in nature $\left(\lambda_{S}>0\right.$ and $\left.\lambda_{C}=0\right)$ and attrition to NATO forces was negligible. The model indicates that under such conditions the rebels could no longer be defeated. The rebels were also provided training and gear to help them repel Qaddafi's attempts at recapturing rebelling population, ie, increasing the rebels' $r_{S}$ but not changing $r_{C}$. Thus, the rebels remained inferior to the regime' forces when fighting in territory controlled by Qaddafi or supporting him. Consistent with our model, we saw a stalemate because Blue victory requires a reduction of $r_{C}$ to $r_{C}<(S) /$ $(1-S)=2 / 3$ (Equation (3)). Finally in the third stage, the foreign intervention gradually began to aid the rebel offensive: NATO provided weapons (decreasing $r_{C}$ ) as well as tactical air support (increasing $\lambda_{C}$ ) (Fahim and Mazzetti, 2011). The near-complete victory by the rebels (as of September 2011) is exactly what one expects from the model when $r_{C}$ is brought sufficiently low.

\section{Afghanistan}

One can view the ongoing conflict in Afghanistan (2001-) as a struggle of government and coalition forces (Blue) against Salafists (Red) led by the Taliban. Many observers of the conflict point to the critical need of both parties to win the support of the population, and thus the conflict is a good application of our model. According to the 2007 report by the International Council on Security and Development, the Taliban have permanent presence in $54 \%$ of the country (International Council on Security and Development, 2007). Suppose, pessimistically, that the Taliban movement has the support of all the people in the regions where it is present. Assuming fixed behaviour of the population (no opportunistic shifts) the situation in Afghanistan will continue in its current stalemate form unless $r_{S}<1.17$ (giving Red a victory) or $r_{C}<0.85$ (giving Blue a victory). Thus, the model suggests that the government can avoid a Taliban takeover of the country by nurturing the support of the population it currently controls, and it is not necessary to push back the Taliban from their areas.

\section{Syria}

The situation in Syria is currently (September 2011) in a state of civil unrest rather than a full-scale armed revolt: The Syrian army and paramilitaries face massive but largly unarmed demonstrations. If the situation does escalate into an armed revolt, what might be its outcome? The opposition (Red) would be at a disadvantage against the government forces (Blue), which are led by special units of the Syrian army that possess superior tactics and weapons. Therefore, $r_{S}$ is likely large and $r_{C}$ is likely small. Furthermore, the international community appears less 
likely to become involved as compared to Libya. However, if the opposition unites under effective leadership and initiates a strong offensive push (perhaps drawing in foreign intervention), then we anticipate that the Assad regime would face a strong challenge because of its narrow base of support in the Alawite sect (assuming he does not enlarge his base).

Recall that Red would be victorious against Blue if $r_{S}<(1-S) /(S)$ (Equation 4). Assuming $S \approx 10 \%$ (the entire Alawite community (Central Intelligence Agency, 2009)), the government must have $r_{S} \geqslant 9$ in order to avoid defeat. If we assume other minority groups (eg, Christians and Druze) also generally support the government (New York Times, 2011), then $S \approx 25 \%$ (Central Intelligence Agency, 2009) and to avoid defeat the government would need just $r_{S} \geqslant 3$. Thus, for the government to avoid defeat in an armed revolt, it would need to maintain strong loyalty of its backers and/or tactical superiority. This seems unlikely: there appears to be strong discontent even within the Alawite community and the rebels could come to acquire military hardware from their foreign backers-hardware such as armor-piercing munitions and air cover to neutralize government forces. Furthermore, the currently observed (September 2011) stalemate is partly a result of foreign intervention by Iranian and Hezbollah combatants (Tisdall, 2011). This intervention is covert and therefore fragile. In sum, although the current situation does not appear promising for the opposition, changes in the domestic political climate, or the international community's stance on intervention could quickly turn the tide and lead to the defeat of Syria's Assad regime.

\section{Summary and conclusions}

We present a new Lanchester-type model that represents the dynamics of liberating and subjugating populated regions in the setting of an armed revolt. We identify winning and stalemate conditions and obtain some general insights regarding the revolt's end-state. Many revolts do not have a decisive outcome, with both sides entrenched in a prolonged stalemate. Our model explicitly identifies this realistic outcome, which is not captured in classical Lanchester theory. Our model also illustrates that it is not sufficient to ably control friendly regions; for victory it is crucial to be able to effectively fight in hostile regions.

We also study the effect of foreign intervention (eg, NATO intervention in Libya) on the outcome of a revolt. We find that although direct intervention to support one side will prevent defeat of that side and can facilitate a win even if that side has very little popular support, indirect intervention cannot guarantee this particularly when its LSER is low. The level of foreign intervention (either direct or indirect) required to defeat an opponent depends on the popular support $(S)$ and the attrition coefficients
$\left(f_{C}\right.$ and $\left.h_{C}\right)$ in the contrarians' territory; it does not depend on the capabilities of the forces in supporters' regions.

Finally, if the population can shift its support, then a stalemate is not possible. A bandwagon-type effect will occur where the population increases its support to the apparent winner, which strengthens it and leads to more support, which further strengthens it and so on until the side achieves victory. Unlike the case of fixed population behaviour, the results of this scenario are sensitive to the initial conditions.

The model we present agrees in its predictions with the views of many analysts of the ongoing conflicts. Therefore, our contribution to the current policy debate is to make explicit the latent assumptions of previous studies. For the future, the model (and its variants) could help to anticipate the outcomes of different kinds of revolts.

\section{References}

Blank L, Enomoto C, Gegax D, McGuckin T and Simmons C (2008). A dynamic model of insurgency: The case of the War in Iraq. Peace Economics, Peace Science and Public Policy 14(2): 1.

Central Intelligence Agency (2009). The World Factbook. U.S. Government online: Washington, DC.

Deitchman SJ (1962). A Lanchester model of guerrilla warfare. Operations Research 10(6): 818-827.

Engel JH (1954). A verification of Lanchester's law. Journal of the Operations Research Society of America 2: 163-171.

Fahim K and Mazzetti M (2011). Rebels' assault on Tripoli began with careful work inside. The New York Times, 22 August, http://www.nytimes.com/2011/08/23/world/africa/23reconstruct .html, accessed 4 October 2011.

Hammes TX (2006). Countering evolved insurgent networks. Military Review 86(4): 18-26.

Hartley DS and Helmbold RL (1995). Validating Lanchester's square law and other attrition models. Naval Research Logistics (NRL) 42(4): 609-633.

Hung CY, Yang GK, Deng PS, Tang T, Lan SP and Chu P (2005). Fitting Lanchester's square law to the Ardennes campaign. The Journal of the Operational Research Society 56(8): 942-946.

International Council on Security and Development (2007). Stumbling into chaos: Afghanistan on the brink, http://www .icosgroup.net/2007/report/stumbling-into-chaos/, accessed 26 May 2011.

Intriligator MD and Brito DL (1988). A predator-prey model of guerrilla warfare. Synthese 76: 235-244.

Johnson IR and MacKay NJ (2011). Lanchester models and the Battle of Britain. Naval Research Logistics (NRL) 58(3): 210-222.

Kress M and Szechtman R (2009). Why defeating insurgencies is hard: The effect of intelligence in counterinsurgency operations - a best-case scenario. Operations Research 57(3): 578-585.

Lanchester FW (1916). Aircraft in Warfare: The Dawn of the Fourth Arm. Constable and Company Ltd: London, UK.

Lucas TW and Turkes T (2004). Fitting Lanchester equations to the battles of Kursk and Ardennes. Naval Research Logistics (NRL) 51(1): 95-116.

Lynn JA (2005). Patterns of insurgency and counterinsurgency. Military Review 85(4): 22-27.

MacAskill E, Watt N, Black I, Pilkington E and Harding L (2011). Libya crisis: Britain, France and US prepare for air strikes 
against Gaddafi. The Guardian, 17 March, http://www.guardian .co.uk/world/2011/mar/17/libya-no-fly-zone-united-nations, accessed 4 October 2011.

McCormick G and Giordano FR (2002). The dynamics of insurgency. Naval Postgraduate School Report.

New York Times (2011). Fearing change, many Christians in Syria back Assad. The New York Times, 27 September, http://www .nytimes.com/2011/09/28/world/middleeast/fearing-change-syriachristians-back-bashar-al-assad.html, accessed 4 October 2011.

Sarkees MR and Wayman F (2010). Resort to War: 1816-2007. CQ Press: Washington, DC, The full database is available at http:// www.correlatesofwar.org, accessed 26 May 2011.

Schaffer MB (1968). Lanchester models of guerrilla engagements. Operations Research 16(3): 457-488.

Small M and Singer JD (1982). Resort to Arms: International and Civil War, 1816-1980. Sage: Beverly Hills.

Strogatz S (2000). Nonlinear Dynamics and Chaos: With applications to Physics, Biology, Chemistry, and Engineering (Studies in nonlinearity). Westview Press: Boulder, Colorado.

Themnér L and Wallensteen P (2011). Armed conflict, 1946-2010. Journal of Peace Research 48(4): 525-536.

Tisdall S (2011). Iran helping Syrian regime crack down on protesters, say diplomats. The Guardian, 9 May, http://www .guardian.co.uk/world/2011/may/08/iran-helping-syrian-regimeprotesters, accessed 4 October 2011.

Washburn A and Kress M (2009). Combat Modeling. International Series in Operations Research and Management Science. Springer Verlag: New York.

\section{Appendix A}

\section{Victory conditions}

In general, dynamical systems may exhibit stable oscillations. We now show that the system of equations in Equation (1) does not have those oscillations (no limit cycles). This means that the state variables will always reach one of the equilibrium points (by the Poincare-Bendixon Theorem (Strogatz, 2000) and noting that the state space is bounded).

Theorem 1 (Dulac's Criterion (Strogatz, 2000) Let $\dot{x}=f(x)$ be a smooth system on a simply-connected set $S \subset \mathbb{R}^{2}$. Let $w: S \rightarrow \mathbb{R}$ be smooth on $S$. Suppose on $S$ the expression $\nabla \cdot(f(x) w(x))$ does not change sign. Then the system has no limit cycle on $S$

Proposition 1 The system of Equation (1) does not have limit cycles.

Proof We first write the model as a system of two independent equations by removing the mixed variables:

$$
\begin{gathered}
S B^{\prime}=+f_{S} S B(S-S B)-h_{S} C R \cdot S B \\
C R^{\prime}=+f_{C} C R(C-C R)-h_{C} S B \cdot C R
\end{gathered}
$$

Let

$$
w(S B, C R)=\frac{1}{S B(S-S B) C R(C-C R)} .
$$

Both the system and $w$ are smooth on the set $(0, S) \times(0, C)$ (points on the boundary of this space move to one of the fixed points and do not oscillate.)

$$
\begin{aligned}
\nabla & \cdot(f w) \\
& =\nabla \cdot\left(\frac{f_{S}(S-S B)-h_{S} C R}{(S-S B) C R(C-C R)}, \quad \frac{f_{C}(C-C R)-h_{C} S B}{S B(S-S B)(C-C R)}\right) \\
& =\frac{-h_{S}}{(S-S B)^{2}(C-C R)}+\frac{-h_{C}}{(S-S B)(C-C R)^{2}}<0 .
\end{aligned}
$$

We next show that the victory conditions in Equations 3,4 correspond to the stability conditions for the equilibria points. Throughout, we assume that $0<S<1$, ie, $S$ is not on its boundary.

Theorem 2 The following four statements hold for the system of differential equations defined by Equation (1):

- The equilibrium $C R=S B=0$ and $S R=1-C B=S$ is never stable.

- The Blue victory equilibrium $(C R=S R=0$ and $S B=1-C B=S)$ is stable if and only if $r_{C}<(S) /(1-S)$.

- The Red victory equilibrium $(S B=C B=0$ and $C R=1-S B=1-S)$ is stable if and only if $r_{S}<(1-S) /(S)$.

- The stalemate equilibrium (defined by Equation (5)) is stable if and only if $r_{C} \geqslant(S) /(1-S)$ and $r_{S} \geqslant(1-S) /(S)$.

Proof The model is fully specified based on two variables: $S B$ and $C R$, Equations (A.1)-(A.2). We first compute the Jacobian of the right hand size of differential equation

$$
\begin{aligned}
& \mathbf{J}(S B, C R) \\
& \quad=\left(\begin{array}{ll}
f_{S}(S-2 S B)-h_{S} C R & -h_{S} S B \\
-h_{C} C R & f_{C}(1-S-2 C R)-h_{C} S B
\end{array}\right)
\end{aligned}
$$

A solution $\left(S B^{*}, C R^{*}\right)$ to the differential equation is stable if the two eigenvalues of $\mathbf{J}\left(S B^{*}, C R^{*}\right)$ have negative real parts (Strogatz, 2000). By inspection the equilibrium with $S B=C R=0$ is not stable for any parameter values.

Blue victory

The characteristic polynomial in this case is

$$
\left(-f_{S} S-\lambda\right)\left(f_{C}(1-S)-h_{C} S-\lambda\right)=0 .
$$

The first eigenvalue, $-f_{S} S$, is always negative and the second eigenvalue, $f_{C}(1-S)-h_{C} S$, is negative if $r_{C}<(S) /$ $(1-S)$. 


\section{Red victory}

The characteristic polynomial in this case is

$$
\left(-f_{C}(1-S)-\lambda\right)\left(f_{S} S-h_{S}(1-S)-\lambda\right)=0 .
$$

The first eigenvalue, $-f_{C}(1-S)$, is always negative and the second eigenvalue, $f_{S} S-h_{S}(1-S)$, is negative if $r_{S}<(1-S) /(S)$. By the dominance assumption (ie, In Equation 2)) $r_{S} r_{C}>1$ and thus $1 /\left(1+r_{S}\right)<\left(r_{C}\right) /\left(1+r_{C}\right)$. Therefore it is not possible for both the Blue victory and Red victory to be stable equilibria for the same values of $r_{S}$ and $r_{C}$. This also implies that if $r_{S}<(1-S) /(S)$ a Blue victory cannot occur and if $r_{C}<(S) /(1-S)$ a Red victory cannot occur.

\section{Stalemate}

At the stalemate equilibrium, the variables have the values $S B_{b}, S R_{b}, C R_{b}$, and $C B_{b}$. We next present a lemma, and then prove that the stalemate equilibrium is stable if and only if $1 /\left(1+r_{S}\right)<S<\left(r_{C}\right) /\left(1+r_{C}\right)$, which is equivalent to the two conditions $r_{C} \geqslant(S) /(1-S)$ and $r_{S} \geqslant(1-S) /(S)$.

Lemma 1 The product $S B_{b} C R_{b}$ is positive if and only if $1 /\left(1+r_{S}\right)<S<\left(r_{C}\right) /\left(1+r_{C}\right)$.

Proof By Equation (5), $S B_{b}$ is positive if $1 /\left(1+r_{S}\right)<S$, and $C R_{b}$ is positive if $S<\left(r_{C}\right) /\left(1+r_{C}\right)$. By the dominance assumption (In Equation 2) $1 /\left(1+r_{S}\right)<\left(r_{C}\right) /\left(1+r_{C}\right)$, and therefore it is impossible for $S B_{b}$ and $C R_{b}$ to both be negative.

Lemma 1 and Equation (5) imply that if $1 /$ $\left(1+r_{S}\right)<S<\left(r_{C}\right) /\left(1+r_{C}\right)$, then $S B_{b}, S R_{b}, C R_{b}$, and $C B_{b}$ are all positive (and by conservation of total population, less than 1). The Jacobian matrix for the stalemate equilibrium is

$$
\mathbf{J}\left(S B_{b}, C R_{b}\right)=\left(\begin{array}{ll}
-f_{S} S B_{b} & -h_{S} S B_{b} \\
-h_{C} C R_{b} & -f_{C} C R_{b}
\end{array}\right)
$$

We derive the upper left hand element of this Jacobian below

$$
\begin{aligned}
\mathbf{J}_{11}\left(S B_{b}, C R_{b}\right)= & f_{S}\left(S-2 S B_{b}\right)-h_{S} C R_{b} \\
= & h_{S}\left(r_{S}\left(S-2 S B_{b}\right)-r_{S} S R_{b}\right) \\
= & f_{S}\left(S-\frac{2 S r_{C}\left(1+r_{S}\right)-2 r_{C}}{r_{S} r_{C}-1}\right. \\
& \left.-\frac{r_{C}-S\left(1+r_{C}\right)}{r_{S} r_{C}-1}\right) \\
= & -f_{S} S B_{b}
\end{aligned}
$$

The lower right hand element of $\mathbf{J}\left(S B_{b}, C R_{b}\right)$ can be derived in a similar fashion and we omit the details. The eigenvalues of $\mathbf{J}\left(S B_{b}, C R_{b}\right)$ will both have a negative real component if the trace of $\mathbf{J}\left(S B_{b}, C R_{b}\right)$ is negative and the determinant is positive The determinant of $\mathbf{J}\left(S B_{b}, C R_{b}\right)$ is $S B_{b} C R_{b} h_{C} h_{S}\left(r_{S} r_{C}-1\right)$. If $1 /\left(1+r_{S}\right)<S<\left(r_{C}\right) /\left(1+r_{C}\right)$, then by Lemma 1 the trace is negative and the determinant is positive, and thus the stalemate equilibrium is stable. If $S \notin\left(1 /\left(1+r_{S}\right),\left(r_{C}\right) /\left(1+r_{C}\right)\right)$ then by Lemma 1 the determinant of $\mathbf{J}\left(S B_{b}, C R_{b}\right)$ is negative and thus one of the eigenvalues has a positive real component and the stalemate equilibrium is not stable.

\section{Appendix B}

\section{Direct foreign intervention}

Let us rewrite the direct intervention dynamics of Equation (6):

$$
\begin{aligned}
& S B^{\prime}=+f_{S}\left(S B+\frac{\lambda_{S}}{f_{S}}\right) S R-h_{S} C R \cdot S B \\
& S R^{\prime}=-f_{S}\left(S B+\frac{\lambda_{S}}{f_{S}}\right) S R+h_{S} C R \cdot S B \\
& C R^{\prime}=+f_{C} C R \cdot C B-h_{C}\left(S B+\frac{\lambda_{C}}{h_{C}}\right) C R \\
& C B^{\prime}=-f_{C} C R \cdot C B+h_{C}\left(S B+\frac{\lambda_{C}}{h_{C}}\right) C R
\end{aligned}
$$

Furthermore let us define $A_{S} \equiv \lambda_{S} / f_{S}$ and $A_{C} \equiv \lambda_{C} / h_{C}$. The stalemate equilibrium of these equations is denoted with a subscript $f i$ (foreign intervention) and we present them below.

$$
\begin{gathered}
S B_{f i}=\frac{S B_{b}}{2}-\frac{A_{S}}{2}+\frac{A_{C}-A_{S}}{2\left(r_{S} r_{C}-1\right)} \\
+\sqrt{\left(\frac{S B_{b}}{2}-\frac{A_{S}}{2}+\frac{A_{C}-A_{S}}{2\left(r_{S} r_{C}-1\right)}\right)^{2}+\frac{r_{S} r_{C} A_{S} S}{r_{S} r_{C}-1}} \\
S R_{f i}=S-S B_{f i} \\
C B_{f i}=\frac{S B_{f i}+A_{C}}{r_{C}} \\
C R_{f i}=1-S-C B_{f i}
\end{gathered}
$$

$\left(S B_{b}\right.$ is the value at stalemate of the variable $S B$ in the basic model, Equation (5).) To derive these expressions we first write the analogue of Equations (A1)-(A.2):

$$
\begin{gathered}
S B^{\prime}=+f_{S}\left(S B+A_{S}\right)(S-S B)-h_{S} C R \cdot S B \\
C R^{\prime}=+f_{C} C R(1-S-C R)-h_{C}\left(S B+A_{C}\right) C R
\end{gathered}
$$


Solving Equations (B.6)-(B.7) for the stalemate equilibrium root results in two equations

$$
\begin{gathered}
f_{S}\left(S B+A_{S}\right)(S-S B)=h_{S} C R \cdot S B \\
f_{C}(1-S-C R)=h_{C}\left(S B+A_{C}\right)
\end{gathered}
$$

Solving Equation (B.8) for $C R$ and substituting into Equation (B.9) produces a quadratic in $S B$. Solving for the positive root of that quadratic yields the expression for $S B_{f i}$ in Equation (B.2). Substituting $S B_{f i}$ into Equation (B.9) gives $C B_{f i}$ in Equation (B.4). Similarly to the basic model, it is possible to exclude cycles, as follows.

Proposition 2 The set of Equation (6) does not have limit cycles.

Proof We will work with the two independent Equations (B.6) and (B.7).

Let

$$
w(S B, C R)=\frac{1}{\left(S B+A_{S}\right) C R(1-S-C R)} .
$$

Both the system and $w$ are smooth on the set $(0, S) \times(0,1-S)$.

$$
\begin{gathered}
w S B^{\prime}=\frac{f_{S}\left(S B+A_{S}\right)(S-S B)-h_{S} C R \cdot S B}{\left(S B+A_{S}\right) C R(1-S-C R)} \\
=\frac{f_{S}(S-S B)}{C R(1-S-C R)}-\frac{h_{S}}{(1-S-C R)}\left(1+\frac{A_{S}}{S B}\right)^{-1} \\
w C R^{\prime}=\frac{f_{C} C R(1-S-C R)-h_{C}\left(S B+A_{C}\right) C R}{\left(S B+A_{S}\right) C R(1-S-C R)} \\
=\frac{f_{C}}{S B+A_{S}}-\frac{h_{C}\left(S B+A_{C}\right)}{\left(S B+A_{S}\right)(1-S-C R)} .
\end{gathered}
$$

Note that $1-S-C R=C-C R>0$ in the strictly positive quadrant. Therefore,

$$
\begin{aligned}
\nabla \cdot(f w)= & \frac{-f_{S}}{C R(1-S-C R)}-\frac{h_{S}}{(1-S-C R)} \\
& \times(-1)\left(1+\frac{A_{S}}{S B}\right)^{-2} \frac{-A_{S}}{S B^{2}} \\
& +0-\frac{h_{C}\left(S B+A_{C}\right)}{\left(S B+A_{S}\right)(1-S-C R)^{2}}<0 .
\end{aligned}
$$

Before proceeding to examine the stability properties of the victory equilibrium and the stalemate equilibrium, we note there are two other equilibrium points to the system defined by Equation (6): $C R=0, S B=-\lambda_{S} / f_{S}$ and an equilibrium similar to Equations (B.2)-(B.5), but with $S B_{f i}$ the negative root of the quadratic that produced Equation (B.2). Because both of these equilibria consist of negative values, which cannot be realized, we do not analyse them further. We next show that the victory condition defined in corresponds to the stability conditions for the equilibria points.

Theorem 3 For the system of differential equations defined by Equation (B.1), the Blue victory equilibrium $(C R=S R=0)$ is stable if and only if $\left(r_{C}-A_{C}\right) /\left(1+r_{C}\right)<S$.

Proof We first compute the Jacobian of the right hand size of differential equation defined in Equations (B.6)-(B.7)

$$
\begin{aligned}
& \mathbf{J}_{\mathrm{fi}}(S B, C R) \\
& \quad=\left(\begin{array}{ll}
f_{S}\left(S-2 S B-A_{S}\right)-h_{S} C R & -h_{S} S B \\
-h_{C} C R & f_{C}(1-S-2 C R)-h_{C}\left(S B+A_{C}\right)
\end{array}\right)
\end{aligned}
$$

For the Blue victory equilibrium $(S B=S, C R=0)$, the characteristic polynomial is

$$
\left(-f_{S}\left(S+A_{S}\right)-\lambda\right)\left(f_{C}(1-S)-h_{C}\left(S+A_{C}\right)-\lambda\right)=0 .
$$

The first eigenvalue, $-f_{S}\left(S+A_{S}\right)$, is always negative and the second eigenvalue, $f_{C}(1-S)-h_{C}\left(S+A_{C}\right)$, is negative if $\left(r_{C}-A_{C}\right) /\left(1+r_{C}\right)<S$. Writing out this condition in terms of the direct foreign intervention parameter $\lambda_{C}=A_{C} h_{C}$ produces the condition: $\lambda_{C}>f_{C}(1-S)+h_{C} S$.

Theorem 3 provides a necessary condition for Blue victory. We have not been able to prove the stability characteristics for the stalemate equilibrium analytically, which would provide the sufficient conditions for Blue victory. This is given in Conjecture 1 and extensive numerical experimentation makes us confident that this conjecture does hold.

Conjecture 1 The stalemate equilibrium defined in Equations (B.2)-(B.5) is stable if and only if $\left(r_{C}-A_{C}\right)$ $\left(1+r_{C}\right)>S$.

\section{Appendix C}

\section{Opportunistic population}

We next show that victory is the only possible outcome of the opportunistic population model. 
Theorem 4 The following four statements hold for the system of differential equations defined by Equation (7):

- The equilibrium $C R=S B=0$ and $S=1 / 2$ is never stable.

- The Blue victory equilibrium $(C R=0$ and $S B=S=1)$ is always stable.

- The Red victory equilibrium ( $S B=S=0$ and $C R=1$ ) is always stable.

- The stalemate equilibrium defined in Equations (8)-(10) is never stable.

Proof We first compute the Jacobian of Equation (7)

$$
\mathbf{J}_{o p}(S B, C R, S)=\left(\begin{array}{l}
f_{S}(S-2 S B)-h_{S} C R \\
-h_{C} C R \\
\alpha
\end{array}\right.
$$

A solution $\left(S B^{*}, C R^{*}, S^{*}\right)$ to the differential equation is stable if the three eigenvalues of $\mathbf{J}_{o p}\left(S B^{*}, C R^{*}, S^{*}\right)$ have negative real parts. By inspection the equilibrium with $\left(S B^{*}, C R^{*}, S^{*}\right)=(0,0,(1 / 2))$ is not stable for any parameter values.

\section{Blue victory}

For the equilibrium $\left(S B^{*}, C R^{*}, S^{*}\right)=(1,0,1)$ the characteristic polynomial is

$$
\left(-h_{C}-\lambda\right)\left(\lambda^{2}+\lambda\left(2 \alpha+f_{S}\right)+\alpha f_{S}\right)=0 .
$$

The first eigenvalue, $-h_{C}$, is always negative and the second and third eigenvalues are always negative by appealing to the quadratic formula for the second term.

\section{Red victory}

For the equilibrium $\left(S B^{*}, C R^{*}, S^{*}\right)=(0,1,0)$ the characteristic polynomial is

$$
\left(-h_{S}-\lambda\right)\left(\lambda^{2}+\lambda\left(2 \alpha+f_{C}\right)+\alpha f_{C}\right)=0 .
$$

The first eigenvalue, $-h_{S}$, is always negative and the second and third eigenvalues are always negative by appealing to the quadratic formula for the second term.

\section{Stalemate}

Substituting the equilibrium points Equations (8)-(10) into the Jacobian yields

$$
\begin{aligned}
\mathbf{J}_{o p}\left(S B^{*}, C R^{*}, S^{*}\right) & \\
= & \left(\begin{array}{lll}
-\frac{f_{S} r_{C}}{2+r_{S}+r_{C}} & -\frac{h_{S} r_{C}}{2+r_{S}+r_{C}} & \frac{f_{S} r_{C}}{2+r_{S}+r_{C}} \\
-\frac{h_{C} r_{S}}{2+r_{S}+r_{C}} & -\frac{f_{C} r_{S}}{2+r_{S}+r_{C}} & -\frac{f_{C} r_{S}}{2+r_{S}+r_{C}} \\
\alpha & -\alpha & -2 \alpha
\end{array}\right)
\end{aligned}
$$

$$
\left.\begin{array}{ll}
-h_{S} S B & f_{S} S B \\
f_{C}(1-S-2 C R)-h_{C} S B & -f_{C} C R \\
\alpha & -2 \alpha
\end{array}\right)
$$

To calculate the eigenvalues, we need to find the roots of the characteristic polynomial $g(\lambda)=\operatorname{det}\left(\mathbf{J}_{o p}\left(S B^{*}, C R^{*}\right.\right.$, $\left.\left.S^{*}\right)-I\right)$. Because $\lim _{\lambda \rightarrow \infty} g(\lambda)=-\infty$, if $g(0)=\operatorname{det}\left(\mathbf{J}_{o p}\left(S B^{*}\right.\right.$, $\left.\left.C R^{*}, S^{*}\right)\right)>0$, then by the Intermediate Value Theorem there must be a positive eigenvalue and the stalemate equilibrium must be unstable. We will now show that this is the case for all possible parameter values. Computing the determinant:

$$
\begin{aligned}
& \operatorname{det}\left(\mathbf{J}_{o p}\left(S B^{*}, C R^{*}, S^{*}\right)\right) \\
& =\alpha\left(-2 f_{S} r_{C} f_{C} r_{S}+h_{S} r_{C} f_{C} r_{S}+f_{S} r_{C} h_{C} r_{S}\right. \\
& \frac{\left.+f_{S} r_{C} f_{C} r_{S}+f_{S} r_{C} f_{C} r_{S}+2 h_{S} r_{C} h_{C} r_{S}\right)}{\left(2+r_{S}+r_{C}\right)^{2}} \\
& =\frac{\alpha r_{S} r_{C}\left(h_{S} f_{C}+f_{S} h_{C}+2 h_{S} h_{C}\right)}{\left(2+r_{S}+r_{C}\right)^{2}}>0 \text {. }
\end{aligned}
$$

Therefore there will always be a positive eigenvalue associated with the stalemate equilibrium defined by Equations (8)-(10), and so it cannot be stable.

Received August 2011; accepted October 2011 after one revision 